\title{
Simulação de sessão tutorial com novos estudantes de medicina de uma universidade
}

\author{
Tutorial session simulation with new medicine students \\ of a universtity
}

\author{
Nathalia Gabay Pereira', Caio César Chaves Costa', Anna Luiza Melo Machado', \\ Juan Monteiro da Silva' \\ 'Centro de Ciências Biológicas e da Saúde, Universidade do Estado do Pará, Belém (PA), Brasil.
}

\section{RESUMO}

Contextualização: A Aprendizagem Baseada em Problemas consiste em um método pedagógico voltado para a autoaprendizagem, com características que proporcionam a aquisição ativa do conhecimento e a formação acadêmica centrada no aluno. Recentemente, tal metodologia faz parte das orientações das Diretrizes Curriculares Nacionais da Graduação em Medicina. No entanto, dificuldades relacionadas à sua execução são ainda bastante comuns. Baseado nessa conjuntura, o presente trabalho busca relatar a experiência de um evento de capacitação na Aprendizagem Baseada em Problemas oferecida para calouros de medicina de uma universidade brasileira. Descrição da Experiência: O programa de capacitação foi executado por meio da simulação de sessões tutoriais. Os novos discentes foram divididos em grupos de discussão, contendo alunos veteranos responsáveis pelo direcionamento da dinâmica da metodologia ativa. Inicialmente, houve a abertura, onde cada conjunto obteve um caso-problema com tema específico, sendo orientados a debater acerca do mesmo e formular objetivos para o estudo independente. Nessa etapa, regras de comportamento dentro da ABP foram estabelecidas e ensinadas aos novos graduandos. Após o término da abertura, os alunos possuíram um período de 4 dias para pesquisa e aquisição do conhecimento até o dia do fechamento tutorial, onde os objetivos foram alcançados e nova discussão acerca do tema foi realizada. Considerações Finais: Apesar das dificuldades iniciais de adaptação, a proposta de capacitação foi considerada satisfatória pelos organizadores do evento, visto que permitiu a inserção dos novos acadêmicos, bem como o desenvolvimento do raciocínio crítico de aprendizado proposto pelas metodologias ativas de ensino.

Recebido: Ago. 07, 2017 Aceito: Set. 15, 2017

\section{COMO CITAR ESTE ARTIGO}

Pereira NG, Costa CCC, Machado ALM, Silva JM. Simulação de sessão tutorial com novos estudantes de medicina de uma universidade. Interdisciplinary Journal of Health Education. 2018 Jan-Dez;3(1-2):10-15 https://doi.org/10.4322/ijhe.2018.005

\section{CORRESPONDÊNCIA}

Caio César Chaves Costa

Universidade do Estado do Pará Tv. Perebebuí, 2623, Marco, CEP 66087-662, Belém (PA), Brasil ccesar.costa@gmail.com

\section{FONTE DE FINANCIAMENTO} Nenhuma.

\section{CONFLITO DE INTERESSE}

Os autores declararam não

haver conflitos de interesse.

O estudo foi realizado no Centro de Ciências Biológicas e da Saúde, Universidade do Estado do Pará, Belém (PA), Brasil.
PALAVRAS-ChAVE: Educação médica. Aprendizagem Baseada em Problemas. Avaliação educacional.

\section{ABSTRACT}

Contextualization: Problem-Based Learning (PBL) consists of a educational method focused on self-teaching, with characteristics that provide the active acquisition of knowledge and the student-centered academic formation. Recently, such methodology is part of the Brazilian National Curricular Guidelines for Medical Graduation; however, difficulties related to its execution are still quite common. Based on this conjuncture, the present work aims to report the experience of a training event in the PBL offered for recent medical students of a Brazilian university. Description of the experience: The training program was executed through the simulation of tutorial sessions. The new students were divided into discussion groups, containing veteran academics responsible for directing the dynamics of the active methodology. Initially, at the opening, each set obtained a problem-case with a specific theme, being oriented to discuss about it and formulate objectives for the independent study. At this stage, rules of behavior within the PBL were established and taught to new graduates. After the opening, the students had a period of 4 days for research and knowledge acquisition until the day of the tutorial shutdown, where the objectives were solved and a new discussion about the theme was performed. Final considerations: Despite the initial difficulties of adaptation, the training proposal was considered satisfactory for the organizers of the event, since it allowed the insertion of the new academics, as well as the development of the critical reasoning proposed by the active teaching methodologies. KEYWORDS: Medical education. Problem-Based Learning. Educational measurement. 


\section{Contextualização}

AAprendizagem Baseada em Problemas (ABP) consiste em um método educacional voltado para o auto-ensinamento ${ }^{1}$. Dentro de sua dinâmica metodológica, os alunos são direcionados à discussão de casos-problema específicos, com posterior formulação de objetivos de estudo orientados à solução do tema proposto ${ }^{2}$.

No interior de instituições de ensino médico, sua performance objetiva o desenvolvimento de raciocínio crítico, bem como de habilidade técnica e analítica para a resolução de problemas práticos na vida real ${ }^{3,4}$. Devido ao fato de que a obtenção do conhecimento ocorre principalmente pelo estudo independente e auto-direcionado do aluno, a ABP se configura como uma rica estratégia educacional para a capacitação e formação dentro da graduação de medicina ${ }^{5}$.

Diversas pesquisas revelam que, quando comparada ao método convencional de ensino, a ABP auxilia na obtenção de maior interesse pelo aprendizado, melhor compreensão dos assuntos e maior independência na estratégia de estudo e busca de informações por parte dos graduandos ${ }^{6-8}$. Estudos de auto-avaliação também denotam alta satisfação dos estudantes acerca do método ${ }^{9,10}$, principalmente, devido ao fato do aprendizado ser mais restrito a discussões com pequenos grupos ${ }^{11}$.

No entanto, inevitavelmente, existem pontos negativos acerca da dinâmica curricular da ABP. Dentre eles, destacam-se o custo para sua implementação e exercício ${ }^{12}$, bem como a maior carga horária e quantidade necessária de professores atuantes ${ }^{13}$. Além disso, requer capacitação profissional por parte dos tutores e orientadores envolvidos, principalmente no que tange ao incentivo, discussão e formulação de estratégias de aprendizado ${ }^{2,4}$. Tais características, quando mal desempenhadas, contribuem para a baixa eficiência do método e estão diretamente relacionadas a falhas na aquisição de conhecimentos e temas médicos específicos em relação à forma tradicional ${ }^{14,15}$.

No Brasil, as diretrizes curriculares de medicina se pronunciam a favor da incorporação de novas metodologias ativas de ensino. Dentro de suas orientações recentes, destaca-se que os regulamentos pedagógicos devem ser centrados no aluno, sendo caracterizados no professor como facilitador e mediador do processo de aprendizagem ${ }^{16}$. À vista disso, as instituições brasileiras de ensino médico estão cada vez mais adeptas à admissão da $\mathrm{ABP}$ como eixo curricular principal, fato este que instala um processo de reformulação da educação em medicina, com características que busquem a independência intelectual do estudante e a indivisibilidade entre teoria e prática ${ }^{17}$.

Como qualquer mudança, a implantação da $\mathrm{ABP}$ dentro da dinâmica pedagógica de universidades e faculdades médicas brasileiras vem sendo acompanhada de dificuldades e problemas relacionados à sua estruturação. Dentre estes, revelam-se a qualificação ineficaz dos docentes e a desorganização dos componentes e eixos de ensino ${ }^{18,19}$. Somado a isso, evidencia-se também a inexperiência por parte dos discentes no processo de aceitação, inserção e desempenho dentro da metodologia ativa ${ }^{20}$, situação que contribui diretamente para a qualidade do processo de graduação.

Nesse contexto, baseado na perspectiva que a ABP deve proporcionar a integração do estudante dentro de seu próprio método de aquisição de conhecimento, propõe-se que a capacitação precoce acerca da dinâmica de sessões tutoriais seja um recurso vantajoso para eficácia posterior do processo de educação médica ${ }^{20}$, fato que é pouco descrito na literatura. Dessa forma, o presente trabalho teve por objetivos promover aos calouros do curso de medicina uma capacitação prática de uma sessão tutorial, bem como relatar a experiência e o impacto dessa capacitação.

Na universidade onde a capacitação foi realizada, a problemática inicial relacionada à $\mathrm{ABP}$ estava relacionada ao desempenho inicial dos novos estudantes nas sessões tutoriais, geralmente expressa por baixo desempenho avaliativo. Adicionalmente, tal fato permitia o crescimento de críticas relacionadas à nova metodologia, bem como da insatisfação por parte dos discentes; justificando-se, principalmente, pela desorganização do método e desqualificação dos profissionais que, em longo prazo, poderiam comprometer o processo de graduação ${ }^{18,19}$. 


\section{Descrição da experiência}

As atividades ocorreram no interior de uma universidade da cidade de Belém, Pará, Brasil; tendo como objetivo capacitar calouros de medicina na dinâmica da Aprendizagem Baseada em Problemas (ABP), exemplo de metodologia ativa requisitada pelas novas Diretrizes Curriculares Nacionais ${ }^{16}$. A participação nas atividades foi de caráter não-obrigatório, sendo estabelecida mediante convite prévio a todos os calouros ingressantes, dos quais 57 aceitaram participar.

O grande estímulo para execução do evento foi baseado na transição metodológica de ensino. Na literatura, é descrito que estudantes habituados com a estratégia tradicional de aprendizado (tal qual a vigente no Ensino Médio), geralmente apresentam dificuldades de adaptação na $\mathrm{ABP}^{21}$. Na maioria dos casos, a problemática se resume em mau desempenho durante as primeiras sessões tutoriais e avaliações somativas, sendo um fator intensificador de ineficiência educacional. Nesse mesmo contexto, cursos preparatórios ${ }^{22}$, bem como a capacitação precoce dos estudantes acerca da dinâmica da $\mathrm{ABP}^{20}$, são ferramentas adequadas para a melhoria dessa metodologia dentro das universidades.

Adicionalmente, o planejamento acadêmico das outras instituições de ensino médico paraenses costuma oferecer apenas um arcabouço teórico introdutório sobre a metodologia ativa, sem espaço para simulações práticas. Portanto, o presente trabalho foi introduzido a partir de uma iniciativa estudantil que buscou proporcionar vivência dinâmica do exercício da $\mathrm{ABP}$ aos calouros, permitindo que pudessem perceber a estruturação ativa do método momentos antes de sua real aplicação.

Para a realização do evento, 21 alunos do $2^{\circ}$ ao $5^{\circ}$ semestre de medicina (voluntários da própria universidade) participaram de treinamentos feitos pelos 5 organizadores, com intuito de que todos se qualificassem e estivessem aptos a interagir e sanar possíveis dúvidas dos novos graduandos a respeito do método pedagógico. Inicialmente, um professor orientador da universidade se responsabilizou em palestrar acerca da metodologia da ABP, apresentando o seu método de aplicação dentro da instituição, bem como a forma de como o planejamento acadêmico estaria inserido dentro das novas diretrizes nacionais. Em seguida, após essa introdução teórica, foi acordado que cada um dos participantes representaria uma função, as quais foram divididas em: (I) Tutor, (II) Estudante-Coordenador e (III) Estudante-Secretário ou Relator; de acordo com o exposto por Borges et al. ${ }^{23}$.

Posteriormente, a todos os veteranos voluntários, foi enviado um caso-problema fictício com tema relacionado ao Sistema Único de Saúde (SUS) e Atenção à Saúde. Além disso, também foram cedidos os objetivos mínimos que os grupos tutoriais deveriam alcançar, bem como planilha com os critérios de avaliação a serem utilizados.

Após essa preparação, houve a abertura do problema tutorial. Os 57 calouros foram divididos em 7 grupos por ordem alfabética. Cada grupo possuía no mínimo 3 veteranos com suas devidas funções previamente estabelecidas (tutor, estudante-coordenador e relator). Ao início da discussão, os calouros foram informados quanto as regras básicas de uma sessão tutorial, tais como: (I) não utilizar aparelhos eletrônicos no momento da troca de informações; (II) não se ausentar da sala de debate; e (III) evitar conversas ou atitudes paralelas à dinâmica, devendo-se manter atenção adequada. Também foram orientados sobre o funcionamento geral de um grupo tutorial, explicando-se qual o compromisso de cada função, a organização da dinâmica e a definição de termos básicos fundamentais, como "objetivos" e "idéias-chave" ${ }^{2}$. Tal iniciação teve duração média de 10 minutos e objetivou instruir os calouros a respeito da execução adequada da ABP. Em seguida, os calouros ficaram livres para a discussão do tema proposto durante período máximo de até 4 horas.

Ao término da abertura do caso-problema, calouros e veteranos elaboraram 3 objetivos mínimos que visavam entender o funcionamento do SUS, bem como os diversos níveis de Atenção à Saúde. Os calouros, então, foram orientados a estudar 
tais objetivos para discutirem posteriormente no dia do fechamento tutorial, onde iriam debater acerca de suas pesquisas e conceitos que haviam aprendido.

Nessa nova etapa, os recém-graduandos possuíram 4 dias para estudar e pesquisar sobre o assunto proposto, colocando em prática a busca ativa de conhecimento preconizada pela $\mathrm{ABP}^{3,4}$. Durante esse tempo, também puderam esclarecer dúvidas entre si e com os veteranos da própria universidade, principalmente relacionadas a estratégias de estudo e auto-aprendizado.

Após 4 dias da abertura, os grupos tutoriais se reuniram novamente para o fechamento do caso-problema. Tal dinâmica possuiu uma duração máxima de 4 horas, sendo direcionada para a resolução dos objetivos e discussão das diferentes fontes de pesquisa do grupo. Ao final da atividade, os participantes ouviram considerações dos veteranos presentes, principalmente acerca dos aspectos positivos e negativos da sessão tutorial e de dicas para melhoria na realização de sua dinâmica nas próximas vezes. Ressalta-se também que nenhum dos calouros foi avaliado com nota, visto que o intuito do evento foi capacitar e explicar a respeito da prática da ABP.

Para o encerramento da atividade proposta, todos os calouros foram reunidos para esclarecimento de suas dúvidas, as quais foram respondidas pelos veteranos voluntários. Ademais, os calouros foram solicitados que escrevessem em papéis acerca de suas percepções sobre a contribuição da simulação para o entendimento da sessão tutorial.

\section{Resultados e impactos}

A maioria dos calouros participantes $(94.7 \%)$ avaliou positivamente a capacitação por meio de anotações recolhidas pelos veteranos do curso, reconhecendo ter aprendido melhor sobre a dinâmica de uma sessão tutorial e considerando-a, nesse primeiro contato, melhor que a abordagem tradicional em virtude da busca independente dos assuntos, que proporcionou maior motivação e aprendizado.

Adicionalmente, observou-se que a introdução dos novos estudantes na $A B P$ motivou a evolução do pensamento crítico antes mesmo do início do curso de fato. Tal estratégia, além de permitir maior aceitação e inserção com o método pedagógico, proporciona a formação de graduandos mais resolutivos e independentes posteriormente ${ }^{24-27}$. Dentre as opiniões oferecidas pelos calouros, destacou-se que o novo método os tornou mais ativos, criando possibilidades para a autoaprendizagem e ampliando a consciência de ser participante influente em sua formação médica.

Entretanto, apesar dos calouros acreditarem que a ABP possui vantagens em relação ao método tradicional, houve dificuldades iniciais de adaptação. A razão principal dada pelos calouros foi exemplificada pelo fato de estarem inseridos em uma nova experiência de ensino, não mais estruturada na forma tradicional observada dentro do ensino médio e fundamental. Como o professor não se comporta como o centro do aprendizado ${ }^{25}$, dúvidas específicas sobre como agir ou se organizar foram freqüentes durante a capacitação. Nesse sentido, o evento serviu como o primeiro encontro, responsável pelo desbloqueio e sequência para outras categorias de instrução.

Outro impacto observado esteve relacionado à grande dificuldade na formulação de hipóteses durante a abertura tutorial, visto que a maioria não apresentava informações prévias para a construção de um bom embasamento teórico. Contudo, no fechamento, foi possível identificar um progresso dentro da discussão, devido os alunos retornarem com maior visão crítica e fontes de pesquisa devidamente consolidadas e confiáveis.

No entanto, após a realização da capacitação, uma parcela dos calouros apresentou maior satisfação durante as tutorias iniciais. Relatos posteriores de 15 calouros indicaram que a simulação prática introdutória permitiu que buscassem mais informações a respeito, bem como se preparassem de forma mais eficiente para o início da programação acadêmica; reduzindo o perfil de descontentamento. Ầ vista 
disso, a iniciativa foi caracterizada como satisfatória para o entendimento, por parte dos calouros, do modelo de aprendizagem proposto pela universidade. Dessa forma, a capacitação foi impulsionada pelos discentes veteranos para que se repetisse nos semestres posteriores.

Tal fato representou o cumprimento do propósito do evento, o qual, apesar das dificuldades, foi capaz de capacitar os estudantes dentro das metodologias ativas. Desde então, a simulação é sempre executada durante as programações iniciais de acolhimento dos calouros.

\section{Considerações finais}

O evento foi considerado satisfatório pelos organizadores, sendo considerado essencial para introduzir os novos estudantes de medicina dentro da dinâmica da $\mathrm{ABP}$, de forma a minimizar possíveis dúvidas posteriores em relação ao método. Além disso, contribuiu para a adaptação inicial dos recém-graduandos, permitindo melhor desempenho avaliativo durante as primeiras sessões tutoriais e maior grau de satisfação em relação à nova metodologia ativa de ensino.

\section{Referências}

1. Leon LB, Onófrio FQ. Aprendizagem baseada em problemas na graduação médica - uma revisão da literatura atual. Rev Bras Educ Med. 2015;39(4):614-9. http://dx.doi.org/10.1590/1981-52712015v39n4e01282014.

2. Wood DF. Problem based learning. BMJ. 2003;326(7384):328-30. http://dx.doi.org/10.1136/bmj.326.7384.328. PMid:12574050.

3. Barrows HS, Tamblyn RM. Problem-based Learning: an approach to medical education. NewYork: Springer; 1980.

4. Borochovicius E, Tortella JCB. Aprendizagem baseada em problemas: um método de ensino-aprendizagem e suas práticas educativas. Ensaio: Avaliação e Políticas Públicas em Educação. 2014;22(83):263-94.

5. Gwee MC. Problem-Based Learning: a strategic learning system design for the education of healthcare professionals in the 21st century. Kaohsiung J Med Sci. 2009;25(5):231-9. http://dx.doi.org/10.1016/S1607-551X(09)70067-1. PMid:19502143.

6. Preeti B, Ashish A, Shriram G. Problem Based Learning (PBL) - an effective approach to improve learning outcomes in medical teaching. J Clin Diagn Res. 2013;7(12):2896-7. PMid:24551668.

7. Zhang Y, Zhou L, Liu X, et al. The effectiveness of the Problem-Based Learning teaching model for use in introductory chinese undergraduate medical courses: a systematic review and meta-analysis. PLoS One. 2015;10(3):e0120884. http:// dx.doi.org/10.1371/journal.pone.0120884. PMid:25822653.

8. Imanieh MH, Dehghani SM, Sobhani AR, Haghighat M. Evaluation of problem-based learning in medical students' education. J Adv Med Educ Prof. 2014;2(1):1-5. PMid:25512911.

9. Abu-Hijleh MF, Kassab S, Al Shboul Q, Ganguly PK. Evaluation of the teaching strategy of cardiovascular system in a problembased curriculum: student perception. Adv Physiol Educ. 2004;28(2):59-63. http://dx.doi.org/10.1152/advan.00010.2003. PMid:15149961.

10. Al-Drees AA, Khalil MS, Irshad M, Abdulghani HM. Students' perception towards the problem based learning tutorial session in a system-based hybrid curriculum. Saudi Med J. 2015;36(3):341-8. http://dx.doi.org/10.15537/smj.2015.3.10216. PMid:25737178.

11. McLean M, Van Wyk JM, Peters-Futre EM, Higgins-Opitz SB. The small group in problem-based learning: more than a cognitive 'learning' experience for first-year medical students in a diverse population. Med Teach. 2006;28(4):e94-103. http://dx.doi.org/10.1080/01421590600726987. PMid:16807164.

12. Albanese MA, Mitchell S. Problem-Based Learning: a review of literature on its outcomes and implementation issues. Acad Med. 1993;68(1):52-81. http://dx.doi.org/10.1097/00001888-199301000-00012. PMid:8447896.

13. Hamdy H, Agamy E. Is running a Problem-Based Learning curriculum more expensive than a traditional Subject-Based Curriculum? Med Teach. 2011;33(9):e509-14. http://dx.doi.org/10.3109/0142159X.2011.599451. PMid:21854146.

14. Millan LPB, Semer B, Rodrigues JMS, Gianini RJ. Traditional learning and problem-based learning: self-perception of preparedness for internship. Rev Assoc Med Bras. 2012;58(5):594-9. http://dx.doi.org/10.1590/S0104-42302012000500018. PMid:23090232.

15. Onyon C. Problem-based learning: a review of the educational and psychological theory. Clin Teach. 2012;9(1):22-6. http:// dx.doi.org/10.1111/j.1743-498X.2011.00501.x. PMid:22225888.

16. Brasil. Ministério da Educação. Conselho Nacional de Educação. Câmara de Educação Superior. Resolução CNE/CES $n^{\circ}$ 3, de 20 de junho de 2014. Institui diretrizes curriculares nacionais do curso de graduação em medicina. Diário Oficial da União, Brasília, DF, 23 jun 2014. Seção 1, p. 8-11.

17. Gomes AP, Rego S. Transformação da educação médica: é possível formar um novo médico a partir de mudanças no método de ensino-aprendizagem? Rev Bras Educ Med. 2011;35(4):557-66. http://dx.doi.org/10.1590/S0100-55022011000400016.

18. Almeida EG, Batista NA. Desempenho docente no contexto PBL: essência para aprendizagem e formação médica. Rev Bras Educ Med. 2013;37(2):92-201. http://dx.doi.org/10.1590/S0100-55022013000200006. 


\section{ijhe}

19. Dolmans DH, De Grave W, Wolfhagen IH, van der Vleuten CP. Problem-Based Learning: future challenges for educational practice and research. Med Educ. 2005;39(7):732-41. http://dx.doi.org/10.1111/j.1365-2929.2005.02205.x. PMid:15960794.

20. Bate E, Taylor DC. Twelve tips on how to survive PBL as a medical student. Med Teach. 2013;35(2):95-100. http://dx.doi. org/10.3109/0142159X.2013.759198. PMid:23383754.

21. McKendree J. Experiences of problem-based learning in the UK. Clin Teach. 2010;7(4):262-5. http://dx.doi.org/10.1111/j.1743498X.2010.00385.x. PMid:21134203.

22. Zaidi Z, Zaidi SM, Razzaq Z, Luqman M, Moin S. Training workshops in problem-based learning: changing faculty attitudes and perceptions in a Pakistani medical college. Educ Health (Abingdon). 2010;23(3):440. PMid:21290363.

23. Borges MC, Chachá SGF, Quintana SM, Freitas LCC, Rodrigues MLV. Aprendizado baseado em problemas. Medicina (Ribeirão Preto). 2014;47(3):301-7. http://dx.doi.org/10.11606/issn.2176-7262.v47i3p301-307.

24. Cyrino EG, Toralles-Pereira ML. Trabalhando com estratégias de ensino-aprendizado por descoberta na área da saúde: a problematização e a aprendizagem baseada em problemas. Cad Saude Publica. 2004;20(3):780-8. http://dx.doi.org/10.1590/ S0102-311X2004000300015. PMid:15263989.

25. Borges TS, Alencar G. Metodologias ativas na promoção da formação crítica do estudante: o uso de metodologias ativas como recurso didático na formação do estudante do ensino superior. Cairu em Revista. 2014;3(4):119-43.

26. Abreu MC, Masetto MT. O professor universitário em aula. São Paulo: MG Editores; 1990.

27. Iwata K, Doi A. Can hybrid educational activities of team and problem based learning program be effective for Japanese medical students? Int J Med Educ. 2017;8:176-8. http://dx.doi.org/10.5116/ijme.591b.2bc8. PMid:28535144.

\section{Contribuição dos autores}

Nathalia Gabay Pereira e Juan Monteiro da Silva contribuíram com a coleta de dados através das entrevistas, análise dos dados e confecção do manuscrito. Caio César Chaves Costa e Anna Luiza Melo Machado contribuíram com a confecção do manuscrito e análise crítica do manuscrito. Nathalia Gabay Pereira e Juan Monteiro da Silva participaram na concepção inicial, acompanhamento, orientação do estudo e elaboração do manuscrito. 